\title{
Primary sutureless repair for "simple" total anomalous pulmonary venous connection: Midterm results in a single institution
}

\author{
Bobby Yanagawa, MD, PhD, ${ }^{\mathrm{a}}$ Abdullah A. Alghamdi, MD, MSc, ${ }^{\mathrm{a}}$ Andreea Dragulescu, MD, \\ Nicola Viola, MD, ${ }^{\mathrm{a}}$ Osman O. Al-Radi, MD, ${ }^{\mathrm{a}}$ Luc L. Mertens, MD, PhD,${ }^{\mathrm{b}}$ John G. Coles, MD, ${ }^{\mathrm{a}}$ \\ Christopher A. Caldarone, MD, ${ }^{\mathrm{a}}$ and Glen S. Van Arsdell, $\mathrm{MD}^{\mathrm{a}}$
}

\begin{abstract}
Objective: We have previously reported the use of an atriopericardial or "sutureless" repair for surgical management of postoperative pulmonary vein stenosis. The potential of avoiding geometric distortion of pulmonary venous suture lines and preventing post-repair pulmonary vein stenosis encouraged us to extend the use of this technique for primary "simple" total anomalous pulmonary venous connection repair.
\end{abstract}

Methods: Between January 1997 and July 2009, 57 consecutive patients (median age, 15 days; median weight, $3.4 \mathrm{~kg}$ ) underwent sutureless or conventional total anomalous pulmonary venous connection repair.

\begin{abstract}
Results: Types of total anomalous pulmonary venous connection included supracardiac in 31 patients (54\%), cardiac in 15 patients $(26 \%)$, and infracardiac in 11 patients $(19 \%)$. Median follow-up time was 2.9 years. Preoperative mean pulmonary vein score, a composite measure of stenosis in all 4 pulmonary veins, was $0.3 / 0-12$, and vertical vein obstruction was found in 35 patients $(61.4 \%)$. A primary sutureless repair was carried out in 21 patients $(36.8 \%$; supracardiac, $\mathrm{n}=12$; cardiac, $\mathrm{n}=4$; infracardiac, $\mathrm{n}=5)$. The sutureless repair group had proportionally greater high-risk infracardiac total anomalous pulmonary venous connection $(24 \%$ vs $16 \%$, $P=.05)$. Primary outcomes of death or reoperation for pulmonary vein stenosis and postoperative pulmonary vein scores $(0.2 \pm 0.7$ vs $0.7 \pm 1.7, P=.26)$ were not different between the techniques.
\end{abstract}

Conclusions: The sutureless repair group had proportionally more infracardiac total anomalous pulmonary venous connection and a higher rate of decline in postoperative right ventricular systolic pressure. Despite increased preoperative risk, no difference was observed in primary outcomes of death and reoperation in the conventional repair group. (J Thorac Cardiovasc Surg 2011;141:1346-54)

Earn CME credits at

http://cme.ctsnetjournals.org

Total anomalous pulmonary venous connection (TAPVC) is a congenital anomaly in which all 4 pulmonary veins do not connect directly to the left atrium. The 2 major risk factors for hospital mortality after surgical repair are pulmonary hypertension and development of pulmonary venous obstruction. Pulmonary venous obstruction can occur at the level of the pulmonary venous confluence to atrial connection or in the pulmonary veins. ${ }^{1,2}$ Reports in the last few years have shown overall hospital mortality ranging from $2 \%$ to $18 \%$ after TAPVC repair. ${ }^{3-7}$ Stenosis of pulmonary venous drainage is reported to occur in $6 \%$ to $9 \%$ of cases after

From the Divisions of Cardiac Surgery ${ }^{\mathrm{a}}$ and Cardiology, ${ }^{\mathrm{b}}$ Labatt Family Heart Centre, the Hospital for Sick Children, University of Toronto, Toronto, Ontario. Disclosures: Authors have nothing to disclose with regard to commercial support.

Read at the 90th Annual Meeting of The American Association for Thoracic Surgery, Toronto, Ontario, Canada, May 1-5, 2010.

Received for publication May 4, 2010; revisions received Sept 3, 2010; accepted for publication Oct 10, 2010; available ahead of print April 1, 2011.

Address for reprints: Glen S. Van Arsdell, MD, 555 University Avenue, Suite 1525, Toronto, ON, M5G 1X8, Canada (E-mail: glen.vanarsdell@ sickkids.ca).

$0022-5223 / \$ 36.00$

Copyright (C) 2011 by The American Association for Thoracic Surgery doi: $10.1016 /$ j.jtcvs.2010.10.056 repair of TAPVC and is particularly prevalent with young age at initial surgery, infracardiac connection type, and preexisting pulmonary vein stenosis (PVS) ${ }^{5,6,8}$ Our group and others have previously described an atriopericardial connection repair for recurrent PVS that uses the pericardium in situ to create a neo left atrium. There are no suture lines on the native pulmonary vein tissue. ${ }^{5,9}$ The repair is referred to as "sutureless" because there is no direct atrial to pulmonary vein or pulmonary venous confluence anastomosis. We use the terms "atriopericardial" repair and "sutureless" repair interchangeably. A potential advantage to this technique is a more limited reactive intimal proliferation because the suture line is not directly on the pulmonary vein. There are also advantages with respect to no direct suture line distortion or narrowing of the veins, particularly if they are small. Optimal flow characteristics for a given vein are therefore intact.

After repair of TAPVC, post-repair pulmonary venous stenosis can occur. Our group previously showed that the use of sutureless repair for post-repair PVS improved survival and reduced the need for reintervention for PVS. ${ }^{10,11}$ Most of our previous discussion on this topic has focused on de novo PVS, after repair of TAPVC, and some primary sutureless repair of TAPVC in the case of right atrial isomerism. A few of the patients presented in this study were briefly mentioned and embedded within 


\section{Abbreviations and Acronyms \\ $\mathrm{ECMO}=$ extracorporeal membrane oxygenation \\ LA $=$ left atrial \\ LAVi = indexed left atrial volume \\ mPAP $=$ mean pulmonary artery pressure \\ PVS = pulmonary vein stenosis \\ RVSP = right ventricular systolic pressure \\ $\mathrm{TAPVC}=$ total anomalous pulmonary venous connection}

a previous article on effectiveness of sutureless repair for various pathology of the pulmonary veins. ${ }^{11}$ The current report focuses on the primary use of an atriopulmonary connection for "simple" TAPVC.

Our initial "primary use" of sutureless repair was in 1998 in a case of infracardiac TAPVC with severe obstruction and a small pulmonary venous confluence. After conventional repair, a residual mean gradient of $2 \mathrm{~mm} \mathrm{Hg}$ was found in all 4 pulmonary veins that did not resolve with revision. The child required extracorporeal membrane oxygenation (ECMO) support and could not be weaned 6 days later because of high mean pulmonary artery pressure (mPAP). The anastomosis was revised to a sutureless technique, and the patient was weaned from cardiopulmonary bypass with resolution of the gradient. Since that time, we have used an atriopericardial connection for all infracardiac TAPVCs. Our practice technique with regard to supracardiac and some cardiac TAPVCs, with mild obstruction at the connection of the confluence to the coronary sinus, has been evolving.

The rationale for an atriopericardial connection is to eliminate potential distortion or narrowing of the pulmonary veins for optimal flow characteristics for a given vein or confluence. Because post-repair pulmonary venous obstruction has been effectively managed with an atriopericardial connection, a second rationale is that it might prevent the development of post-repair PVS. We present our midterm results comparing outcomes of the atriopericardial procedure with a conventional repair for a subset of "simple" TAPVC during an interval of practice evolution. Outcomes with respect to mortality and development of pulmonary venous obstruction are evaluated.

\section{PATIENTS AND METHODS \\ Patient Data}

Data on patients with isolated TAPVC were collected at the Hospital for Sick Children, University of Toronto, between January 1997 and July 2009. Those with the following conditions were excluded: associated congenital cardiac lesions, such as right atrial isomerism and hypoplastic left heart syndrome, mixed-type TAPVC, syndromic, and single ventricle. We identified 57 consecutive patients with "simple" TAPVC. Ethics approval from our institutional review board was obtained. Patient demographic, operative and postoperative records, clinic notes, and echocardiographic imaging were studied. Baseline patient characteristics are listed in Table 1 . The median age at time of initial surgical intervention was 15 days (range, $1-1157$ days) and median weight was $3.4 \mathrm{~kg}(1.7-11.7 \mathrm{~kg})$. Median follow-up was 2.9 years (range, 5 days to 11.7 years). Patients from earlier study years (1997-2004) were more likely to be repaired primarily with a conventional approach, and patients from later study years (2005-2009) were more likely to be repaired primarily with a sutureless repair (Figure 1).

\section{Pulmonary Vein Score}

The pulmonary vein score for each individual vein was calculated as previously reported. ${ }^{11}$ Briefly, pre- and postoperative echocardiographic data were reviewed to quantify the degree of PVS: $0=$ no stenosis (mean gradient $<2 \mathrm{~mm} \mathrm{Hg}$ ); 1 = mild stenosis (mean gradient 2.0-6.9 $\mathrm{mm} \mathrm{Hg}$ ) 2 = severe stenosis (mean gradient $>7 \mathrm{~mm} \mathrm{Hg}$ ); and $3=$ complete occlusion. The sum of the individual pulmonary vein scores is then used as a subjective measure of the overall degree of PVS ranging from 0 (no stenosis) to a theoretic maximum score of 12 .

\section{Echocardiographic Measurements}

Echocardiographic images were evaluated in a blinded manner for type of repair and outcome. Right ventricular systolic pressure (RVSP) was evaluated using the tricuspid regurgitant jet, and mPAP was estimated from the early diastolic velocity of the pulmonary regurgitant jet. For both measurements, the modified Bernoulli equation was used. Left ventricular ejection fraction was measured from M-mode or 2-dimensional measurements of left ventricular end-diastolic and end-systolic dimensions using the Teichholz formula. Indexed left atrial volume (LAVi) was measured as $0.85^{*}$ left atrial (LA) area in apical 4-chamber view*LA area in apical 2-chamber view/LA length/body surface area, as described previously. ${ }^{12}$ The collecting chamber was included in the LA volumetric measurement in the postoperative study. Mean gradients of the Doppler traces of the individual pulmonary veins were also measured.

\section{Statistical Analysis}

Data are presented as frequencies, medians with ranges, or means with standard deviations. Survival data are represented by the Kaplan-Meier survival curve. Longitudinal data analysis was performed using mixedeffects models for analysis and to generate a graphic display of the averaged trend lines for the echocardiographic data. Statistical analyses were performed using SAS (Version 9.1; SAS Institute Inc, Cary, NC) and R (Version 2.10, R Project for Statistical Computing).

\section{Surgical Technique}

Surgical repair was performed on cardiopulmonary bypass (mean time, $88.0 \pm 31.0$ minutes) with bicaval cannulation. For cardiac TAPVC $(n=15$ in total), the coronary sinus was unroofed in the conventional repair group $(\mathrm{n}=11)$ and further included single- or 2-sided atriopericardial connection in the sutureless repair group $(n=4)$ if there was associated vein to confluence orifice stenosis. For sutureless repair of infracardiac and supracardiac TAPVC, incisions were made in the venous confluence and then extended into both upper and lower pulmonary veins separately if judged to be important for unobstructed flow (Figure 2). Small pulmonary venous confluences (ie, smaller than the normalized mitral valve size diameter), such as those seen in infracardiac TAPVC, were more likely to require incisions in the individual pulmonary veins out toward the pleural pericardial reflection. An atriopericardial anastomosis was created using the pericardium adjacent to the pulmonary vein entrance to the pericardium. A neo left atrium was thus created. The atriopericardial anastomosis avoids direct contact with the vein wall incision site and allows the free egress of blood from the lungs into the left atrium (Figure 2). The atrial septal defect or patent foramen ovale was closed or partially closed. Other details have been described in our previous report. ${ }^{11}$ 
TABLE 1. Patient preoperative, operative, postoperative, and outcomes data

\begin{tabular}{|c|c|c|c|c|c|c|c|c|}
\hline \multirow{2}{*}{$\begin{array}{c}\text { Variable } \\
\text { Preoperative }\end{array}$} & \multicolumn{4}{|c|}{ Conventional repair } & \multicolumn{4}{|c|}{ Sutureless repair } \\
\hline & Overall & Supracardiac & Cardiac & Infracardiac & Overall & Supracardiac & Cardiac & Infracardiac \\
\hline $\mathrm{N}$ & 36 & 19 & 11 & $6^{*}$ & 21 & 12 & 4 & $5^{*}$ \\
\hline Age (d) & $19(1-1157)$ & $21(0-387)$ & $46(0-1157)$ & $3(1-19)$ & $11(1-138)$ & $5.5(0-72)$ & $64(11-138)$ & $20(1-23)$ \\
\hline Weight (kg) & $3.5(1.7-11.7)$ & $3.4(2.4-6.5)$ & $3.6(2.7-11.7)$ & $3.1(1.7-4.1)$ & $3.3(1.9-5)$ & $3.5(2.3-5.1)$ & $3.0(1.9-4.4)$ & $3.2(2.7-3.9)$ \\
\hline PVS & $0.2 \pm 1$ & $0.3 \pm 1.4$ & $0.0 \pm 0.0$ & $0.2 \pm 0.4$ & $0.5 \pm 1.2$ & $0.3 \pm 1.2$ & $1.5 \pm 1.9$ & $0.0 \pm 0.0$ \\
\hline Male (\%) & 55 & 52.6 & 45.5 & 45.5 & 57 & 50.0 & 100.0 & 18.2 \\
\hline $\operatorname{LVEF}(\%)$ & $66.3 \pm 10.1 *$ & $70.6 \pm 10.8$ & $61.9 \pm 8.4^{*}$ & $61.8 \pm 5.1^{*}$ & $74.5 \pm 7.2^{*}$ & $72.0 \pm 8.9$ & $76.8 \pm 2.2^{*}$ & $78.0 \pm 4.6^{*}$ \\
\hline LAVi & $5.9 \pm 3.1$ & $5.9 \pm 3.1$ & $7.1 \pm 3.4$ & $3.6 \pm 1.5$ & $4.8 \pm 3.4$ & $4.2 \pm 2.5$ & $8.5 \pm 5.0$ & $2.9 \pm 0.8$ \\
\hline $\begin{array}{l}\text { Vertical vein } \\
\text { obstruction }\end{array}$ & $18(50 \%)^{*}$ & $9(47.4 \%)^{*}$ & $3(27.3 \%)^{*}$ & $6(54.5 \%)^{*}$ & $17(81 \%)^{*}$ & $11(91.7 \%)^{*}$ & $4(100 \%)^{*}$ & $5(45.5 \%)^{*}$ \\
\hline \multicolumn{9}{|l|}{ Operative } \\
\hline CPB time & $84.5 \pm 34.6$ & $98.2 \pm 38.9$ & $69.8 \pm 27.8$ & $72.7 \pm 15.8$ & $93.8 \pm 23.2$ & $84.3 \pm 11.6$ & $111.8 \pm 22.2$ & $100.4 \pm 35.2$ \\
\hline Crossclamp time & $48.8 \pm 22.4^{*}$ & $56.9 \pm 25.9$ & $39.4 \pm 16.0$ & $42.3 \pm 11.5^{*}$ & $62.3 \pm 19.3^{*}$ & $56.5 \pm 17.7$ & $69.8 \pm 23.6$ & $70.6 \pm 18.7 *$ \\
\hline DHCA time & $4.5 \pm 10.9^{*}$ & $0.7 \pm 2.8^{*}$ & $6.2 \pm 14.4$ & $13.2 \pm 15.0$ & $15.9 \pm 19.2^{*}$ & $11.3 \pm 16.0^{*}$ & $5.5 \pm 11.0$ & $35.4 \pm 20.3$ \\
\hline \multicolumn{9}{|l|}{ Postoperative } \\
\hline Pulmonary vein score & $0.7 \pm 1.7$ & $0.3 \pm 1.0$ & $1.0 \pm 1.4$ & $1.3 \pm 3.2$ & $0.2 \pm 0.7$ & $0.3 \pm 1.0$ & $0.0 \pm 0.0$ & $0.7 \pm 1.2$ \\
\hline Mean PAP (mm Hg) & $23.8 \pm 13.7$ & $28.7 \pm 18.7$ & $34.3 \pm 26.6$ & - & $28.3 \pm 12.5$ & $0.0 \pm 0.0$ & - & $22.5 \pm 10.6$ \\
\hline RVSP (mm Hg) & $33.5 \pm 13.3$ & $35.4 \pm 10.0$ & $34.4 \pm 26.6$ & $20.5 \pm 3.5^{*}$ & $40.5 \pm 11.8$ & $42.7 \pm 15.0$ & $35.0 \pm 9.9$ & $40.0 \pm 6.6^{*}$ \\
\hline $\operatorname{LVEF}(\%)$ & $69.3 \pm 6.2$ & $69.9 \pm 6.7$ & $37.0 \pm 5.9$ & $67.3 \pm 6.2$ & $67.1 \pm 9.7$ & $65.0 \pm 11.0$ & $73.0 \pm 7.5$ & $68.6 \pm 6.3$ \\
\hline LAVi & $15.8 \pm 6.5$ & $15.9 \pm 6.3$ & $18.9 \pm 6.9$ & $11.1 \pm 4.2$ & $14.8 \pm 5.7$ & $13.3 \pm 5.2$ & $20.8 \pm 6.2$ & $14.7 \pm 5.1$ \\
\hline $\begin{array}{l}\text { Length of hospital } \\
\text { stay (d) }\end{array}$ & $10(2-195)$ & $13.0(5-75)$ & $9.0(2-195)$ & $16(8-108)$ & $17(7-112)$ & $13.5(7-112)$ & $17.5(7-63)$ & $19(9-60)$ \\
\hline \multicolumn{9}{|l|}{ Outcomes } \\
\hline Follow-up time (d) & $\begin{array}{c}45.8 \\
(0-143.1)^{*}\end{array}$ & $\begin{array}{c}47.0 \\
(0.3-131.1)\end{array}$ & $\begin{array}{c}34.7 \\
(0.3-137.3)\end{array}$ & $\begin{array}{l}53.7 \\
(0-143.1)\end{array}$ & $\begin{array}{c}21.1 \\
(0.2-104.8)^{*}\end{array}$ & $\begin{array}{c}46.8 \\
(1.8-99.7)\end{array}$ & $\begin{array}{l}12.5 \\
(0.2-30.2)\end{array}$ & $\begin{array}{c}19.8 \\
(0.2-104.8)\end{array}$ \\
\hline Reoperation & $2(5.5 \%)$ & $1(4.7 \%)$ & $0(0 \%)$ & $1(16.7 \%)$ & $0(0 \%)$ & $0(0 \%)$ & $0(0 \%)$ & $0(0 \%)$ \\
\hline Cardiac death & $0(0 \%)$ & $0(0 \%)$ & $0(0 \%)$ & $0(0 \%)$ & $0(0 \%)$ & $1(8.3 \%)$ & $1(25 \%)$ & $0(0 \%)$ \\
\hline Noncardiac death & $0(0 \%)$ & $0(0 \%)$ & $0(0 \%)$ & $0(0 \%)$ & $2(9 \%)$ & $0(0 \%)$ & $0(0 \%)$ & $0(0 \%)$ \\
\hline
\end{tabular}

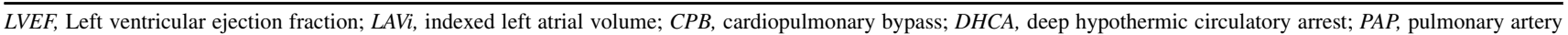
pressure; $P V S$, pulmonary vein stenosis; $R V S P$, right ventricular systolic pressure. ${ }^{*} P<.05$.

\section{RESULTS}

\section{Preoperative}

A total of 57 patients were included in this series, of whom 36 underwent conventional repair and 21 underwent sutureless repair. Preoperative vertical vein stenosis was more common in the sutureless repair group compared with the conventional repair group $(81 \%$ vs $50 \%$, $P=.02$ ). However, pulmonary venous obstruction (pulmo-

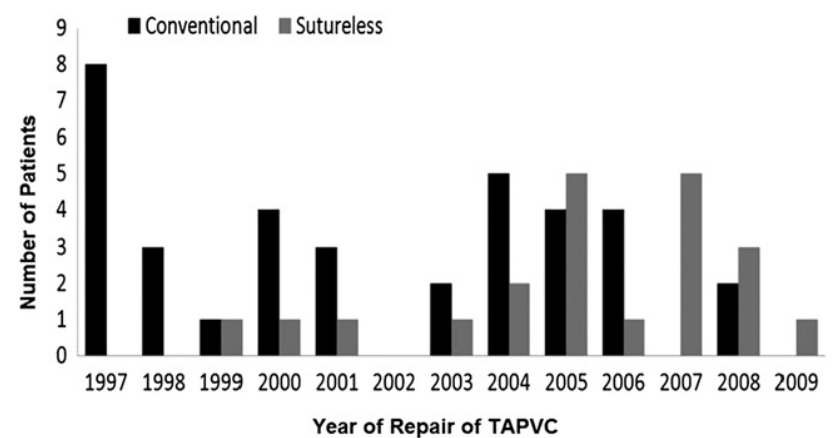

FIGURE 1. Number of conventional versus sutureless repairs performed by year at the Hospital for Sick Children. TAPVC, Total anomalous pulmonary venous connection. nary vein score; $0.5 \pm 1.2$ vs $0.2 \pm 1.0, P=.38)$ and $\mathrm{LAVi}$ were not significantly different between the groups ( $4.8 \pm$ 3.4 vs $5.8 \pm 3.1, P=.25$ ). Other preoperative characteristics were similar between the sutureless and conventional repair groups (Table 1).

\section{Operative}

Total cardiopulmonary bypass time was similar in both groups. However, crossclamp and circulatory arrest times were longer in the sutureless repair group $(P=.02$; Table $1)$. The longer crossclamp and deep hypothermic circulatory arrest times for sutureless repair were most likely related to surgeon bias because the surgeon who performed the majority of sutureless repairs chose to do so with circulatory arrest. When separated into TAPVC types, crossclamp times were longer in infracardiac sutureless repair compared with conventional repair $(70.6 \pm 18.7$ vs 42.3 $\pm 11.5, P<.05)$, which may reflect greater preoperative anatomic complexity.

\section{Postoperative}

Postoperative characteristics were not significantly different between the 2 groups with regard to total pulmonary 


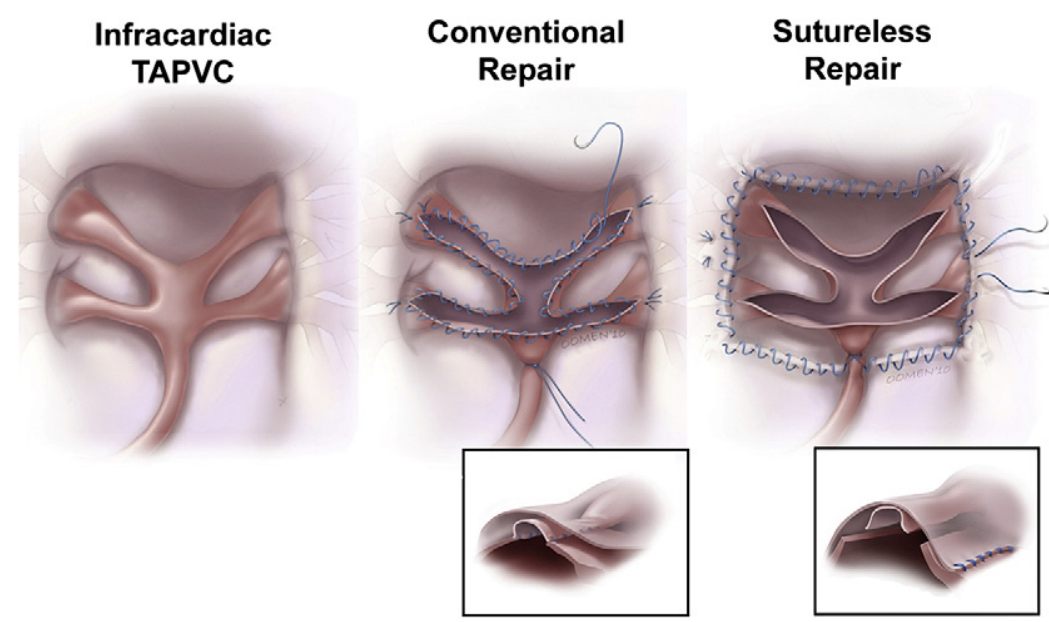

FIGURE 2. Surgical repair for TAPVC. Conventional repair, conventional repair (pulmonary vein insert with potential suture line stenosis), and sutureless repair (pulmonary vein insert) techniques are shown. TAPVC, Total anomalous pulmonary venous connection.

vein score, LAVi, or mPAP. Hospital length of stay was similar (Table 1).

The overall RVSP was not significantly different; however, in the infracardiac repair group, the RVSP was higher in the sutureless repair group $(20.5 \pm 3.5$ vs $40.0 \pm 6.6$, $P<.05$ ), likely reflective of higher grade preoperative obstruction. Averaged trend lines for the longitudinal echocardiographic RVSP are similar between the 2 groups (Figure 3). Multivariable regression analysis revealed that patients with sutureless repair had higher RVSP than average baseline $(P=.003)$, and this decreased at a higher linearized rate than in those with conventional repair $(P=.02)$. Among patients who were selected for sutureless repair, those with cardiac TAPVC had higher RVSP at baseline compared with supracardiac TAPVC $(P=.002)$. The linearized rate of decline in RVSP over time in patients who underwent sutureless repair tended to be slower in patients who underwent infracardiac TAPVC versus cardiac TAPVC $(P=.05)$, and versus supracardiac TAPVC $(P=.02)$. We found no differences in the overall rate of decline in mPAP and ejection fraction percent pre- and postoperatively in both repair groups. There were no significant differences in pulmonary vein score pre- and postoperatively in both repair groups.

\section{Outcomes}

Median follow-up time was $45.8(0-143.1)$ months in the conventional repair group and $21.1(0.2-104.8)$ months in the sutureless repair group. Two patients in the conventional repair group underwent reoperation for PVS (5.5\%; Figure 4). Unilateral pulmonary vein occlusion developed in 1 patient who underwent cardiac TAPVC in the sutureless repair group, but this patient did not undergo reoperation (RVSP 45, mPAP 32). Two patients in the sutureless group

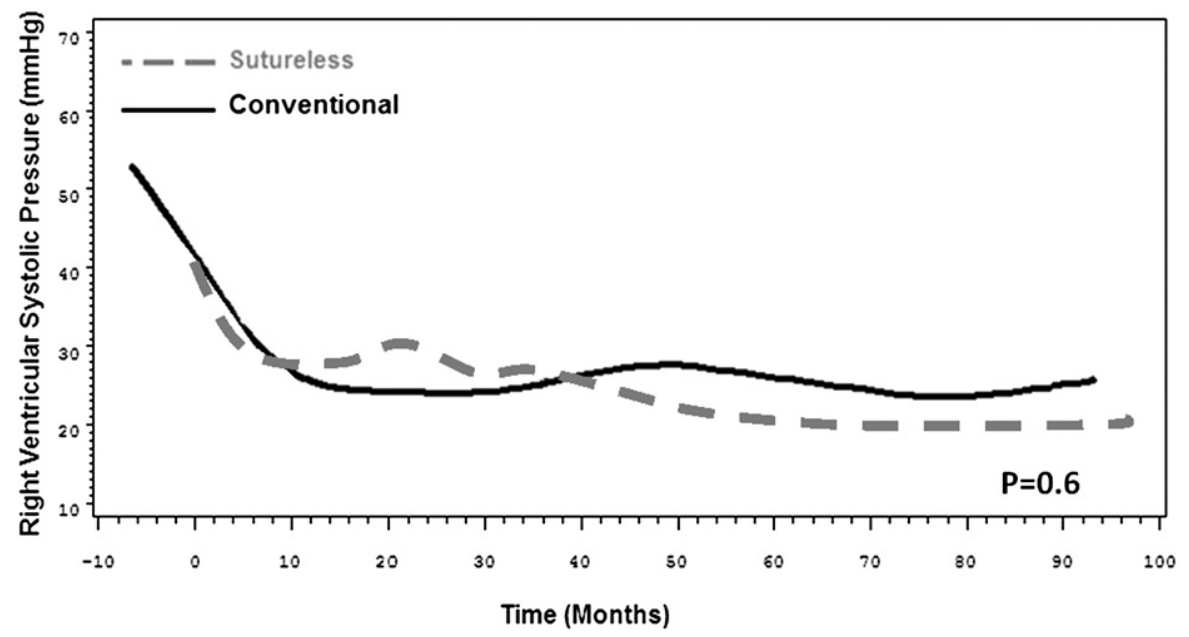

FIGURE 3. Longitudinal averaged trends for RVSP. Patients with TAPVC in the conventional and sutureless repair groups were compared with respect to serial postoperative RVSP. 


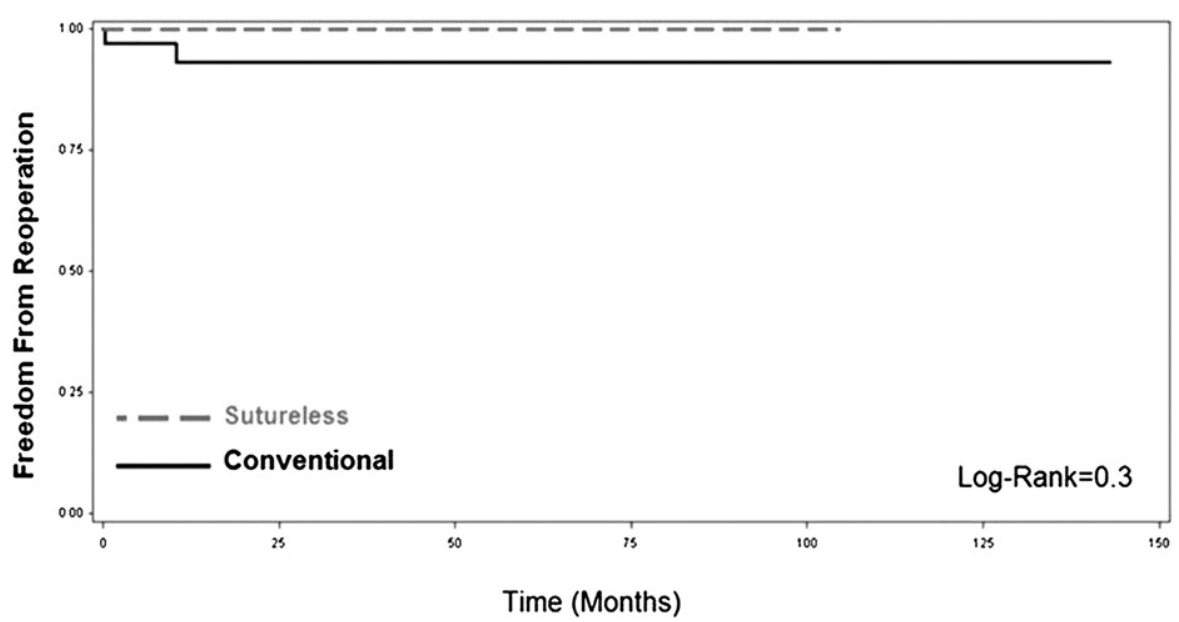

FIGURE 4. Freedom from reoperation. Patients with TAPVC in the conventional and sutureless repair groups were compared with respect to freedom from reoperation.

died: One died postoperatively of necrotizing enterocolitis related to a tracheoesophageal fistula repair, and one died of pulmonary hypertension at 10 months of age, which persisted despite documented nonobstructed pulmonary veins $(3.8 \%)$. One infant in the sutureless repair group required a permanent pacemaker for complete heart block. There were no cardiac causes of death in either repair group in our series. Overall mortality or repair failure (overall death or reoperation) was not statistically significant between the 2 groups (Figure 5).

\section{Comment}

As in most biventricular repair-type congenital anomalies, early mortality has substantially declined for TAPVC. In a subset, early post-repair pulmonary hypertension and lung function still remain a challenge. Late mortality related to pulmonary venous stenosis has not been eliminated. For those with infracardiac TAPVC with very small pulmonary veins and pulmonary venous confluences, achieving a repair that has no distortion or even limited stenosis can be challenging. A direct suture line on very small pulmonary veins or a small confluence can leave distortion or minor gradients (Figure 2). Strategies to address the technical problems of post-repair pulmonary venous obstruction for small confluences and veins have been the creation of a generoussized pulmonary venous confluence to LA anastomosis and the use of interrupted or absorbable sutures that extend into the individual pulmonary veins (Figure 2). Small pulmonary vein gradients remain in some patients and can be a significant physiologic burden because of secondary pulmonary hypertension.

Our exploration of the sutureless repair strategy for obstructed infracardiac TAPVD first occurred in an attempt at a salvage procedure for a failed conventional repair in

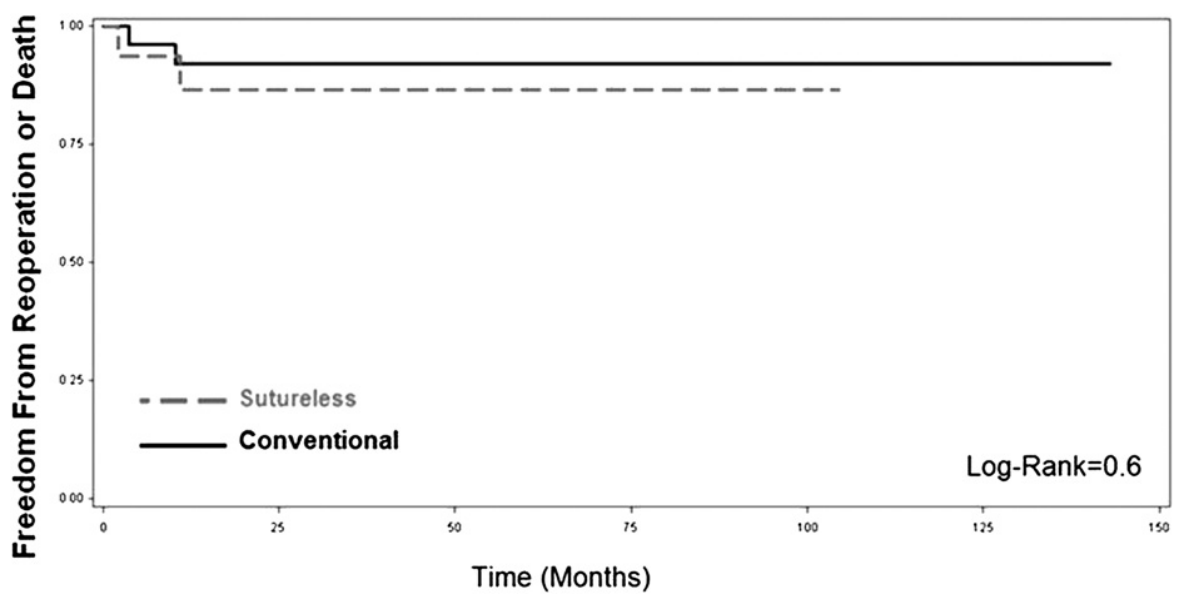

FIGURE 5. Freedom from reoperation or death. Patients with TAPVC in the conventional and sutureless repair groups were compared with respect to freedom from reoperation and all-cause mortality. 
a patient who could not be weaned from ECMO. The successful application of the sutureless technique in that patient and the recognized potential benefits of not having an anastomosis on the small confluence or veins led to a subsequent deliberate strategy to use the technique for small confluences and veins. We rationalized that by eliminating anastomotic distortion to the pulmonary veins, post-repair lung function and early pulmonary hypertension may be better managed. The indications to use the technique in larger veins and confluences were liberalized according to the surgeon's judgment for a better technical outcome. We also observed that the atriopericardial suture line can be technically easier to perform, particularly if the veins or confluence are borderline or small in size.

Because of the low incidence of occurrence for this disease, the total numbers and events in this study are relatively small. The associated small number of event end points leads to little statistical power. By univariate analysis, however, despite a greater proportion of infracardiac TAPVC, there was no demonstrable difference in early and late mortality. Previous data have shown that preoperative pulmonary vein obstruction was a risk factor for mortality and development of late PVS. One might hypothesize, therefore, that the lack of difference in outcomes data for a higher-risk population is favorable. Our current indications for sutureless repair are (1) all infracardiac TAPVC, (2) intracardiac TAPVC with evidence of pulmonary vein stenosis, and (3) surgeon preference for supracardiac TAPVC.

\section{Preoperative and Postoperative Pulmonary Venous Obstruction}

Preoperative pulmonary venous obstruction has been associated with significant mortality in the context of TAPVC. ${ }^{4,6,9,13,14}$ Jenkins and colleagues ${ }^{15}$ reported that individual pulmonary vein diameter and small vein confluence were significant predictors of survival in patients with TAPVC. Although contemporary overall hospital mortality for TAPVC repair ranges from $2 \%$ to $18 \%$, it increases to $30 \%$ to $66 \%$ in the presence of recurrent pulmonary vein stenosis. ${ }^{1,3-7}$ The present study found no difference in pulmonary vein scores between the sutureless and conventional repair groups ( $0.5 / 12$ vs $0.2 / 12)$.

The reported incidence of postoperative PVS varies from $1 \%$ to $18 \%$, with larger series showing an incidence of $6 \%$ to $9 \%{ }^{6,8,14-16}$ A previous study from our institution found reoperation for PVS to be associated with young age at initial surgery, cardiac connection type, and existing pulmonary venous obstruction. ${ }^{11}$ In the present study, 21 patients $(36.8 \%)$ underwent operation within 1 week of birth, 15 patients $(26.3 \%)$ had cardiac connection-type TAPVC, and 5 patients $(8.8 \%)$ had some degree of preoperative PVS.

Two patients in the conventional repair group required reoperation: the index patient and a patient who underwent conventional repair for a large confluence nonobstructed supracardiac TAPVC in whom stenosis developed in the first few months after repair. Unilateral pulmonary vein obstruction with retrograde stenosis into the lung developed in 1 patient in the sutureless group. The pulmonary pressures were mildly elevated. No operation was performed. No patient in this series died of pulmonary venous obstruction.

\section{Pulmonary Hypertension}

Persistent pulmonary hypertension is a common postoperative complication after TAPVC repair, particularly in the setting of pulmonary venous obstruction. ${ }^{17}$ Treatment may include medical therapy, such as nitric oxide, mechanical resuscitation with ECMO or surgical revision, or septostomy. Failed repair or recurrence can lead to mortality or the requirement of lung or heart-lung transplantation. ${ }^{18}$ By multivariate analysis, patients in the sutureless repair group had higher preoperative RVSP, which decreased at a higher rate than in those in the conventional repair group. This is a potential explanation for the ability to manage the pulmonary hypertension without the need for ECMO in recent years. Immediate postoperative RVSP was 2 times higher in the infracardiac TAPVC group with sutureless repair compared with conventional repair despite having lower postoperative pulmonary vein scores. We suspect this occurred because the sutureless repair group had worse preoperative obstruction. Notably, once the policy to use sutureless repair was instituted, we did not require the use of ECMO for pulmonary hypertension in any further patients. With time, the early elevated RVSP in the sutureless repair group was lower than in the conventional repair group, implying that a satisfactory anatomic outcome was achieved.

\section{Left Atrial Size}

We measured LA size, hypothesizing that a more benign postoperative course, with respect to cardiac and pulmonary function issues related to pulmonary hypertension, might be explained by a potential LA enlargement from the atriopericardial connection. No difference in atrial size was demonstrated between the operative strategies.

\section{Potential Risks in Sutureless Repair}

There is potential risk in an atriopericardial repair, including (1) thrombogenicity at the exposed pericardial surface, (2) air embolism, (3) soft tissue rupture, and (4) phrenic nerve injury.

We have anticoagulated some patients using lowmolecular-weight heparin when there was concern of potential clot formation from raw posterior mediastinal tissue that is sometimes exposed and incorporated into the left atrium in an atriopericardial connection. Although the connection is hemostatic, the pulmonary venous confluence is not in direct contact with the suture line. Therefore, hemostasis is dependent on the integrity of the posterior mediastinum and pleural pericardial junction. A breach in the integrity 
of that junction can result in bleeding into the pleural space, a complication that may be managed by intrapleural hilar approximation. ${ }^{11}$ In general, use of primary atriopericardial anastomosis, as opposed to its use for pulmonary venous stenosis, does not require as aggressive an incision into the individual pulmonary veins. The risk of intrapleural bleeding should therefore be less than that seen in de novo pulmonary venous stenosis sutureless repairs.

We have some concern regarding closed-chest compression in the event of need for postoperative cardiopulmonary resuscitation. We would prefer to perform open chest message if cardiopulmonary resuscitation is required. Our rationale is that closed chest compression could result in pleural rupture and lead to atriopleural exsanguination. We believe the risk is low, unless there is an aggressive incision into the subpleural portion of individual pulmonary veins. Phrenic nerve injury and diaphragmatic paralysis are risks. The present cohort did not have phrenic nerve injury.

After assembling these data, we performed a sutureless repair on a neonate with mixed-type obstructive TAPVC. High mean airway pressure oscillation ventilation was required postoperatively. Cardiac arrest necessitated ECMO. At ECMO initiation, air bubbles were found in the aorta, which we suspect was related to mediastinal air induced by the high ventilation pressures. In this particular case, a distal incision into the individual veins within the pulmonary parenchyma had been made because of their small size. The distal incision may have raised the risk of mediastinal air tracking into the left atrium. The child survived without neurologic sequelae. ${ }^{19}$

\section{Limitations}

Limitations of this study are the retrospective nature, small study population, and small number of events. We demonstrate potential trends that will bear further analysis and that may prove to be statistically significant given a larger sample size. Specifically, we found potential trends in higher preoperative pulmonary vein score, RVSP, and LAVi despite lower values after sutureless repair. Finally, there may be an era effect because patients from earlier study years (1997-2004) were repaired primarily with a conventional approach and patients from later study years (2005-2009) were repaired primarily with a sutureless approach.

\section{CONCLUSIONS}

Sutureless repair for TAPVC was effective in relief of pulmonary venous obstruction. Development of late pulmonary venous obstruction was not different between sutureless and conventional repair patients. No patient died of pulmonary venous obstruction. The sutureless repair group had proportionally more infracardiac TAPVC and a higher rate of decline in postoperative RVSP. Despite increased preoperative risk, no difference was observed in primary outcomes of death and reoperation in the conventional repair group, suggesting that application of the technique to high-risk patients may be beneficial. The sutureless technique is a useful addition to the surgeon's armamentarium, particularly for repair of difficult cases, such as infracardiac TAPVC.

\section{References}

1. Hancock Friesen CL, Zurakowski D, Thiagarajan RR, Forbes JM, del Nido PJ, Mayer JE, et al. Total anomalous pulmonary venous connection: an analysis of current management strategies in a single institution. Ann Thorac Surg. 2005; 79:596-606.

2. Caldarone CA, Najm HK, Kadletz M, Smallhorn JF, Freedom RM, Williams WG, et al. Surgical management of total anomalous pulmonary venous drainage: impact of coexisting cardiac anomalies. Ann Thorac Surg. 1998;66:1521-6.

3. Kelle AM, Backer CL, Gossett JG, Kaushal S, Mavroudis C. Total anomalous pulmonary venous connection: results of surgical repair of 100 patients at a single institution. J Thorac Cardiovasc Surg. 2010;139:1387-94. e3. Epub 2010 Apr 14.

4. Lacour-Gayet F. Surgery for pulmonary venous obstruction after repair of total anomalous pulmonary venous return. Semin Thorac Cardiovasc Surg Pediatr Card Surg Annu. 2006;45-50.

5. Caldarone CA, Najm HK, Kadletz M, Smallhorn JF, Freedom RM, Williams WG, et al. Relentless pulmonary vein stenosis after repair of total anomalous pulmonary venous drainage. Ann Thorac Surg. 1998;66:1514-20.

6. Sano S, Brawn WJ, Mee RB. Total anomalous pulmonary venous drainage. J Thorac Cardiovasc Surg. 1989;97:886-92.

7. Lamb RK, Qureshi SA, Wilkinson JL, Arnold J, West CR, Hamilton DI. Total anomalous pulmonary venous drainage: seventeen-year surgical experience. J Thorac Cardiovasc Surg. 1988;96:368-75.

8. Wilson WR Jr, Ilbawi MN, DeLeon SY, Quinones JA, Arcilla RA, Sulayman RF, et al. Technical modifications for improved results in total anomalous pulmonary venous drainage. J Thorac Cardiovasc Surg. 1992;103:861-70.

9. Lacour-Gayet F, Zoghbi J, Serraf AE, Belli E, Piot D, Rey C, et al. Surgical management of progressive pulmonary venous obstruction after repair of total anomalous pulmonary venous connection. J Thorac Cardiovasc Surg. 1999;117:679-87.

10. Najm HK, Caldarone CA, Smallhorn J, Coles JG. A sutureless technique for the relief of pulmonary vein stenosis with the use of in situ pericardium. $J$ Thorac Cardiovasc Surg. 1998;115:468-70.

11. Yun TJ, Coles JG, Konstantinov IE, Al-Radi OO, Wald RM, Guerra V, et al. Conventional and sutureless techniques for management of the pulmonary veins: evolution of indications from postrepair pulmonary vein stenosis to primary pulmonary vein anomalies. J Thorac Cardiovasc Surg. 2005;129:167-74.

12. Lang RM, Bierig M, Devereux RB, Flachskampf FA, Foster E, Pellikka PA, et al. Recommendations for chamber quantification: a report from the American Society of Echocardiography's Guidelines and Standards Committee and the Chamber Quantification Writing Group, developed in conjunction with the European Association of Echocardiography, a branch of the European Society of Cardiology. J Am Soc Echocardiogr. 2005;18:1440-63.

13. Bando K, Turrentine MW, Ensing GJ, Sun K, Sharp TG, Sekine Y, et al. Surgical management of total anomalous pulmonary venous connection. Thirty-year trends. Circulation. 1996;94:II12-6.

14. Yee ES, Turley K, Hsieh WR, Ebert PA. Infant total anomalous pulmonary venous connection: factors influencing timing of presentation and operative outcome. Circulation. 1987;76:III83-7.

15. Jenkins KJ, Sanders SP, Orav EJ, Coleman EA, Mayer JE Jr, Colan SD. Individual pulmonary vein size and survival in infants with totally anomalous pulmonary venous connection. J Am Coll Cardiol. 1993;22:201-6.

16. Kirshbom PM, Myung RJ, Gaynor JW, Ittenbach RF, Paridon SM, DeCampli WM, et al. Preoperative pulmonary venous obstruction affects longterm outcome for survivors of total anomalous pulmonary venous connection repair. Ann Thorac Surg. 2002;74:1616-20.

17. Lupinetti FM, Kulik TJ, Beekman RH, Crowley DC, Bove EL. Correction of total anomalous pulmonary venous connection in infancy. J Thorac Cardiovasc Surg. 1993;106:880-5.

18. Mendeloff EN, Spray TL, Huddleston CB, Bridges ND, Canter CB, Mallory GB Jr. Lung transplantation for congenital pulmonary vein stenosis. Ann Thorac Surg. 1995;60:903-6.

19. Alghamdi AA, Coles JG, Holtby H, Al-Radi OO. Massive air embolism after the repair of obstructed total anomalous pulmonary venous drainage: an unusual complication. J Card Surg. 2010;25:582-4. 


\section{Discussion}

Dr Francois Lacour-Gayet (New York, NY). Primary repair of total abnormal pulmonary venous return using the sutureless technique is extremely appealing, and your experience in Toronto shows excellent results. The conventional repair results in terms of mortality and morbidity have significantly improved. We have found it best to use a technique that does not manipulate the pulmonary vein. Nevertheless, some anatomic forms remain at risk. They include, as you mentioned, the small pulmonary vein confluence in the infracardiac type, the mixed venous forms, and, for an unknown reason, the total vein associated with a single ventricle. I have a few questions.

In view of the good results you have observed, would you consider using the primary sutureless technique for miscellaneous and single ventricle forms, the total pulmonary resistance associated with heterotaxy-type single ventricle?

Dr Alghamdi. In our previously published report, sutureless repair was used as a primary procedure for PVS associated with TAPVD and isomeric atrial arrangement. Most of these patients had right atrial isomerism. Some patients also had a single ventricle. The results were encouraging. These patients were excluded from this cohort.

Dr Lacour-Gayet. One advantage of the technique is to be able to safely open the trunk of the pulmonary vein with minimal risk of stenosis. Do you always incise the pulmonary vein trunks using this technique?

Dr Alghamdi. The extension of the incision of the pulmonary venous confluence to the individual pulmonary veins will be applied if there is documented preoperative PVS or the pulmonary veins are small on the subjective intraoperative assessment. Therefore, extension of the incision to the individual veins is not done routinely.

Dr Lacour-Gayet. I have seen in your series that you report using the technique in the intracardiac form. The application of the technique to intracardiac form seems more challenging because by definition the pulmonary veins are attached to the heart by the width of the coronary sinus. Furthermore, the operation to unroof the coronary sinus and patch from the coronary sinus to the atrial septal defect is, in a way, already a sutureless technique. Would you comment on your experience in applying the sutureless technique to intracardiac types?

Dr Alghamdi. Performing the sutureless technique for the cardiac-type TAPVC is only performed when the unroofing of the coronary sinus is not adequate (ie, when there are documented small pulmonary veins).

Dr Lacour-Gayet. Finally, the occurrence of pulmonary vein obstruction after a repair seems unpredictable and could be in relation to an abnormal metabolism of the native pulmonary venous endothelium. In the situation in which a pulmonary venous obstruction occurs despite a perfect primary sutureless repair, you will be left with no ammunition to repair the pulmonary vein obstruction. Do you consider that this limitation could be a reason for elective primary sutureless repair only to the adverse form?

Dr Alghamdi. Can you repeat that again?

Dr Lacour-Gayet. While considering that once you have done the repair, you cannot do it again, the question is, if it happens despite an excellent surgery, won't you then be left with the only option of giving the patient to the cardiologist? And my question is, for the simple form, knowing that even with an excellent technique you sometimes can have pulmonary vein obstruction, it's not bad to have this ammunition ready.

Dr Alghamdi. PVS will develop in some patients, despite what is considered a "perfect" repair. We propose though, that the sutureless technique minimizes the "trigger" for the development of PVS. Although the sutureless technique does not abolish the risk of PVS, it is associated with a low incidence of PVS, which is a reasonable justification to use it up-front.

Dr Lacour-Gayet. I want to congratulate the group in Toronto for this innovative technique. It will be useful to elective patients.

Dr Peter Manning (Cincinnati, Ohio). Just a quick technical question regarding bleeding complications. In a primary repair such as this, the planes behind the pericardium may not be as well socked in as in a child in whom PVS has developed after a prior operation. One experience that we have had using this technique in a child with infracardiac total veins as a primary procedure was bleeding, where the blood dissected from our newly created space down to the area where we had dissected and ligated the vertical vein. Do you avoid, particularly in the infracardiac cases, dissecting the vertical vein and just leave it patent? In the supracardiacs, do you still ligate the vein? How do you manage or avoid that problem?

Dr Alghamdi. Most of the infracardiac types have obstructed vertical veins anyway, so it would be reasonable to just leave the vertical vein alone. An alternative would be to ligate the vertical vein from the inside of the confluence to avoid dissecting around the vertical vein.

For the supracardiac type, it is not unreasonable to leave the vertical vein alone if it is obstructed. Our approach has been to ligate the vertical vein routinely.

In terms of the other bleeding complications, in a previous report, in those with post-repair stenosis, where the incision of the pulmonary vein extended toward the hilum of the lung, the integrity of the pleuro-pericardial junction was violated, which resulted in bleeding into the pleura space, and that was repaired by approximation of that area between the hilum and the pleura.

Dr Manning. I would imagine in these cases you're probably not opening the veins as far out toward the hilum as you would in a reoperative case as well.

Dr Alghamdi. That's absolutely correct.

Dr Renato Assad (Sao Paulo, Brazil). In regard to an anticoagulation protocol, do you recommend any antithrombotic therapy for those patients undergoing a sutureless procedure for "simple" TAPVC?

Dr Alghamdi. One would think there is a raw surface in the left heart side that may initiate some thromboembolic complications. We do not have a standard protocol to anticoagulate these patients; it is based on the surgeon's discretion. Some surgeons would anticoagulate if they think the area of raw surface is extensive, and others would not. But certainly we haven't seen any thromboembolic complications associated with this primary repair even in the absence of anticoagulation.

Dr Assad. If you administer any antithrombotic therapy, how long would you suggest continuing treatment?

Dr Alghamdi. Nobody knows. But what we do sometimes, and that's again based on the surgeon's discretion, is continue treatment for 6 weeks. 
Dr Assad. In regard to those patients who experienced progressive pulmonary venous obstruction after primary repair, have you given any endothelin-receptor antagonist or chemotherapy after sutureless repair?

Dr Alghamdi. No.

Dr Assad. Is there any protocol with chemotherapy or Persantine for those patients?

Dr Alghamdi. No, not at this time. Thank you!

Dr Zhou Hong (Wuhan, China). I have one question. In our experience, I have had a case of TAPVC with a branch of the PVS. In your opinion, can the sutureless procedure be used in these patients?

Dr Alghamdi. If you had a TAPVC and the veins all drained into the confluence and one of these veins was really small, what we would do is open the confluence and extend the incision of the confluence into the vein itself until it appears healthy and then do the primary sutureless repair.
Dr James Tweddell (Milwaukee, Wis). What if the stenosis extends outside the pericardia?

Dr Alghamdi. As long as that integrity between the pericardium and the pleura is intact, you can extend it toward the hilum of the lung. Extension of such an incision too far is not without potential risks. We have seen a similar patient (not part of this cohort) with mixed-type TAPVD and very small veins. The incision was extended into the hilum of the lungs. When the patient was transferred from the operating room to the intensive care unit, she required high-pressure ventilation because the lungs were very stiff. The patient immediately collapsed and required an ECMO. When the aortic cannula was inserted into the aorta, a significant amount of air was seen inside the aorta in the form of froth. That massive air embolism was possibly due to a communication with the left atrium. The patient was on ECMO for a few days and weaned successfully. She had a remarkable recovery with no significant complications and was discharged. 